\title{
THE DEPOT LEPROMIN TEST AND BCG VACCINATION
}

\author{
J. A. Kinnear Brown, M.I., B.Sc., D.T.M. \\ Specialist Leprologist, Uganda \\ and M. M. STONE, S.R.N., S.C.M.
}

\section{Kumi Ongino Leprosy Settlement, Uganda}

Investigations reported recently suggest that for purposes of preventive medicine weak reactors to normal lepromin could be identified if they can be considered to be those who would fail to respond to a multi-puncture test with $1: 100$ depot lepromin. This test has none of the drawbacks of the ordinary Mitsuda, it is simple to apply, economical of material and would have advantages in field work. (Kinnear Brown 1958). The depot medium used consisted of one part of anhydrous lanoline and eight parts of light liquid paraffin. 0.25 gramme of autoclaved bacteriologically positive lepromatous tissue was ground into $4 \mathrm{ml}$. of medium and $1 \mathrm{ml}$. of isotonic saline to form a suspension in a concentration of $1: 20$. A dilution of 1 : 100 was made by adding medium and saline in similar proportions.

Fourteen healthy children about one year old, whose mothers had leprosy were now tested with both concentrations. One child was positive to both, the others negative. Five weeks later 12 of the 14 were tuberculin tested. They were all negative. Ten were then vaccinated with BCG and retested with $1: 20$ depot lepromin.

The child who was lepromin positive at the beginning remained positive. The nine who were negative became positive. The signs of conversion appeared first in the final lepromin test, in one child in three weeks, in the others after four. The maximum response was reached in four to six weeks. Conversion signs in the original lepromin tests were later by two weeks, when seven of the nine children showed positive in both concentrations; there was some slight alteration at the sites in the other two children but the signs were less definite. In these seven therefore the depot lepromin had remained in the skin long enough to act as an indicator and the final test might have been avoided. It is possible this would have happened in all if the interval between the original tests and the vaccination had been three weeks instead of five; the longer interval was deliberate.

Conversion was not demonstrated by the original lepromin tests for 11 weeks, i.e., six weeks after the BCG vaccination. Had there been any significant sensitisation by the dose of lepromin injected in those tests one would have expected conversion in them not later than in the final tests. Lepromin is a comparatively weak antigen and in the dosage given by the multi-puncture route it is difficult 
to imagine it could have any profound effect except in a very small minority. The order of events suggests that in these children the BCG vaccination was responsible for the conversion.

The work was repeated on a further 13 children who were negative to both lepromin and tuberculin. Nine of the 13 showed conversion at the end of six weeks. It was not possible to follow up the other four, as they went home to relatives, this being the reason why only ten of 14 children in the first series completed the schedule.

By contrast there was no change to positive in 22 lepromin negative patients who were tested and BCG vaccinated, which confirms the impression that the effect of BCG on patients and nonpatients is different.

\section{Conclusion}

I. Depot lepromin injected by the multi-puncture route remained in the skin long enough to act as an indicator.

2. The considerable economy of material that is possible and the saving of time both to the examiner and the individual examined make it desirable that the method should be further investigated with a view to its use in the field and in control campaigns.

\section{Acknowledgements}

Our thanks are due to Dr. J. M. Lea, Medical Superintendent of the Kumi Ongino Leprosy Settlement, for his assistance and giving access to his patients.

\section{References}

Brown, J. A. Kinnear, Leprosy Review, 19, 4: Oct. 1958, pp. 184-196 